\title{
USING MACHINE VISION FOR FUNCTIONALITY EXPANSION OF MINI ROBOTS DECONTAMINATING MEDICAL PERSONNEL PREMISES IN CONDITIONS OF COVID-19 EPIDEMIC
}

\author{
M.M. Knyazkov ${ }^{1 *}$, A.V. Polyakov², V.M. Usov² \\ ${ }^{1}$ Ishlinsky Institute for Problems in Mechanics of the Russian Academy of Sciences (IPMech RAS), Moscow, E-mail: \\ ipm_labrobotics@mail.ru \\ ${ }^{2}$ Russian Federation State Research Center - Institute of Biomedical Problems RAS (IBMP RAS), Moscow, E-mail: \\ apolyakov@imbp.ru
}

KEY WORDS: COVID-19, Monitoring technologies, Automated air environment control, Robotic medical complexes, Service robotics, Technical vision systems.

\begin{abstract}
:
In case of COVID-19 epidemic spread the requirements for protection of medical personnel were increased. This category of specialists has exposure of high risk of COVID-19, due to inevitable numerous contacts with infected persons.

Because of this, existing practicable models of medical care are needed to be upgraded (Tavakoli, 2020). The emergency response path was implemented through the opening of new infectious hospitals, re-profiling clinics, as well as increasing workload on medical personnel. This approach is associated with the possible rapid drop-up of qualified medical specialists due to the illness, which is a strong limiting factor to respond to new threats of COVID-19 due to the risks of exhaustion of the human resource. In the worst scenario, a threat of collapse of the emergency and specialized medical care system due to peak load of severe patients under the shortage of doctors and support personnel. To prevent such an emergency, a complex of anti-epidemic events is provided, most of the purpose of interrupting infection contacts, isolation of the most vulnerable contingents, extended population testing for virus or contact with infected persons, this allows you to follow unwanted contacts with the subsequent mobility limitation.

Digital monitoring technologies with digitization of incoming data of significant events played the increasing role in all these options. Today they are complemented by robotic supporting. Due to the high risk of the infection in the COVID-19 spread, including the intra-clinic infections, the requirements for reliable disinfection of closed premises intended for patients and medical personnel should be fulfilled. At the same time, the following circumstance should be taken into account. When these work-time activities are imposed on the employee who is forced to stay long in an infected air environment, he is subjected to additional impact of pathogenes. To reduce these threats, robotic support for the work for disinfection of premises with automated air environment control and tracing contacts.
\end{abstract}

\section{INTRODUCTION}

As noted above, there are various ways of active opposition to the spread of COVID-19. Among the most preferable ways is the path of reducing infectious load on each person, which leads to the idea of expanding the role of robotic technologies to identify pathogenes in working areas, disinfection of problematic locations, as well as using digital technologies for epidemiological tracking of the most likely infection chains in the visit of these places (Tavakoli, 2020). Until recently, this functionality has not been so much in demand, although there are a number of useful applications of robotic medical complexes (RMC), which help reduce the workload on medical personnel even outside the epidemiological context. When the COVID-19 spread, the resort to such RMC allow to release the medical personnel from auxiliary operations for more important tasks of conducting severe patients. However, for such target as reducing the level of infectious load in problematic locations of the working environment of medical personnel, today the rational way is to modify existing products if they can serve the prototypes of products with anti-epidemic functionality. This requires analytical research, in which it is necessary: (a) to determine the priorities of the development of specific types of RMC and the points of their application; (b) to describe the required functionality of specialized RMC and means of achieving it, taking into account the real state of anti-epidemic toolkit, placed on board robots; (c) to consider the economic side of the mass introduction of such expensive equipment in practical medicine in the conditions of the expected level of financing (according to the developed state programs). A separate position is related to the human factor of implementation of autonomous robots and safety of their functioning in a difficult configurable application environment, especially if it is a question of RMC group. The methodological setting in the systematization of typical approaches and solutions that give a beneficial effect from the anti-epidemic purpose of RMC in practically acceptable terms, proceeded from the current level of knowledge of the subject area - to counteract the spread of COVID-19 by expanding the role of $\mathrm{RMC}$ as a special type of service robotics. The requirements for the functionality of the RMC in the field of health protection of medical personnel, recommended by regulatory documents and methods of epidemiological control and disinfection of the working environment, have been established.

\footnotetext{
* Corresponding author
} 
The purpose of this study was to analyze prototypes from a large number of existing and newly proposed RMC solutions that can claim in the conditions of COVID-19 to achieve the required functionality, safety of use in human society environments, effective use of mounted (or placed on board) tools, taking into account human factors and other advantages according to the criterion of reducing the risk of infection for medical personnel. First of all, the expected effects were supposed to be achieved by solving the problems of detecting coronavirus in the air environment of closed rooms of medical institutions and decontaminating the locations of medical personnel's and patients locations.

\section{SOLVING THE PROBLEMS OF MOBILE ROBOT NAVIGATION WITH TECHNICAL VISION TECHNIQUES}

To prevent collisions that may occur during the interaction of robots with each other or with a human (Figure 1), it is necessary to have onboard navigation sensors and devices that will allow avoiding collisions with a human and any mobile or stationary objects with a high probability degree especially during counteract measures against the COVID-19 pandemic spreading. For indoor spaces disinfection the following two mobile robots platforms are used: on base of UV-radiation disinfection and on liquid antiseptics dispersion. Before SARSCoV-2COVID pandemia these methods were used only within hospitals' premises. However these days it's emerging to use them in heavily concoursed areas, e.g. in trade centers, service centers etc.

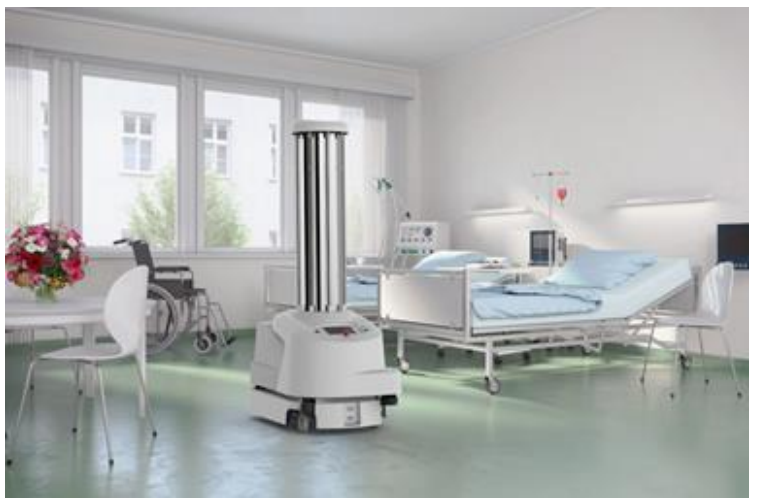

Figure 1. Blue Ocean Robotics MODEL C.

To meet the requirements mentioned above, a computer vision system (CVS) can be used as the main information sensor node, which is provided with TV camera system. CVS essentially performs the functions of the robot's "eyes" and is able to use a TV camera to digitize the image of the surrounding space and to some extent provide data about the physical characteristics of objects located in it in the form of the following characteristics:

— object size;

— location of the object in space;

— overview of the object (color, surface condition, etc.);

— object markers (logos recognition, QR codes, etc.).

However, in conditions where the mobile robot should be used in smoky or dusty space, the data from conventional TV cameras become unreliable, and there is a need to apply specialized ultrasonic or thermal imaging devices that can ensure the performance of the CVS in these conditions. In such conditions, it is possible to use thermal imagers operating in the IR range, which allow assessing the environment in low light conditions and extreme conditions. The thermal imager can be installed on a mobile robot with conventional TV cameras and thus a multi-band CVS will be formed. The use of thermal imagers, however, does not completely eliminate all interference, because the problem of the resulting geometric noise, which is inherent in highly sensitive multi-element thermal radiation sensors, has not been completely solved technologically even until now, which leads to significant distortions of the resulting image. To implement algorithms for constructing the trajectory of movement of robotic systems, it is necessary to have an idea of the location of obstacles relative to the robot, in order to make a map of the surrounding space that the robot observes. To determine the relative position of the robot and the surrounding objects, it is necessary to determine the depth of the image of the environment transmitted from the robot using data analysis. To determine the depth of the picture of the environment, one can use specialized scanning devices, such as a laser rangefinder or LIDAR (Figure 2), and to process the information obtained from them, use the techniques and algorithms of SLAM (Simultaneous Localization and Mapping). However, the use of such equipment will greatly affect the final cost of the mobile robotic system, since it is very expensive.

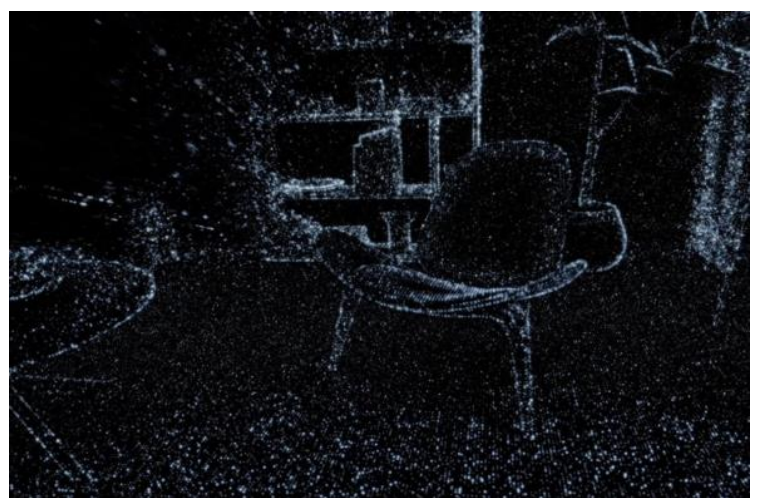

Figure 2. Generated 3D model of the room using the LIDAR scanner.

To solve navigation problems, a stereo vision system can be integrated within the robot (Smirnov, 2016), which will allow estimating the distance to objects and can be used for mapping the environment. Such systems are much cheaper than expensive sonars and LIDARs, since they usually use two cameras that work synchronously relative to each other. The Semi-Global Block-Matching Algorithm (Ryan, Roelofs, 2013) is used to construct environmental maps that allow estimating the depth of the image transmitted from the cameras (Figure 3).

In order to detect obstacles and follow-up image recognition and classification onto static and dynamic structures and also humans' detection stereo vision cameras should be installed (Figure 4). These cameras allow to detect distance to the object (Maryum F. Ahmed, 2006), as well a determine objects' motion parameters (direction, velocity and acceleration)

With the help of CVS, in addition to tasks related to the creation of the robot's route, some tasks should also be solved to recognize QR codes - "tags" that someone can leave in order to eliminate possible interference with the robots (Figure 5). 
In addition, for robots that work directly with people (Figure 6), it is highly desirable to use algorithms that will help with face verification, face identification, age and gender determination, and emotions determination. This will allow robots organically engaging in the work of medical personnel and significantly reduce the risk of uninformed aggression directed at the robot.

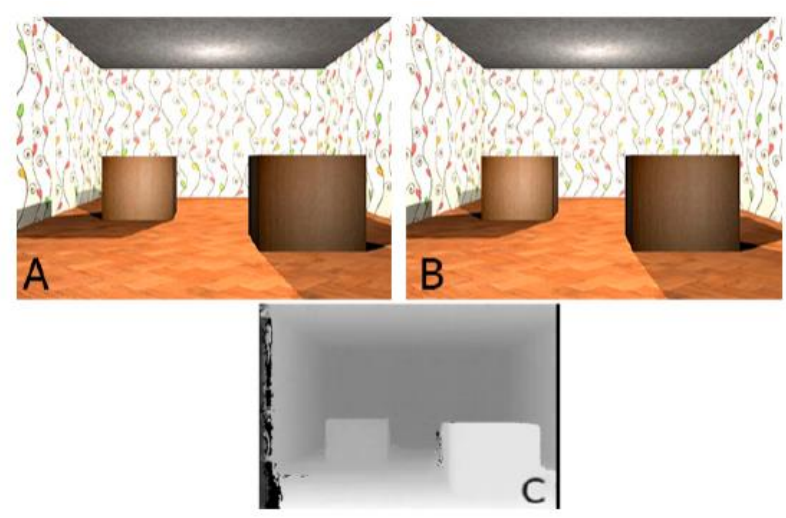

Figure 3. A - left camera image; B - right camera image; C depth map obtained from the virtual environment map.

(Smirnov, Bezzubcev, 2016)

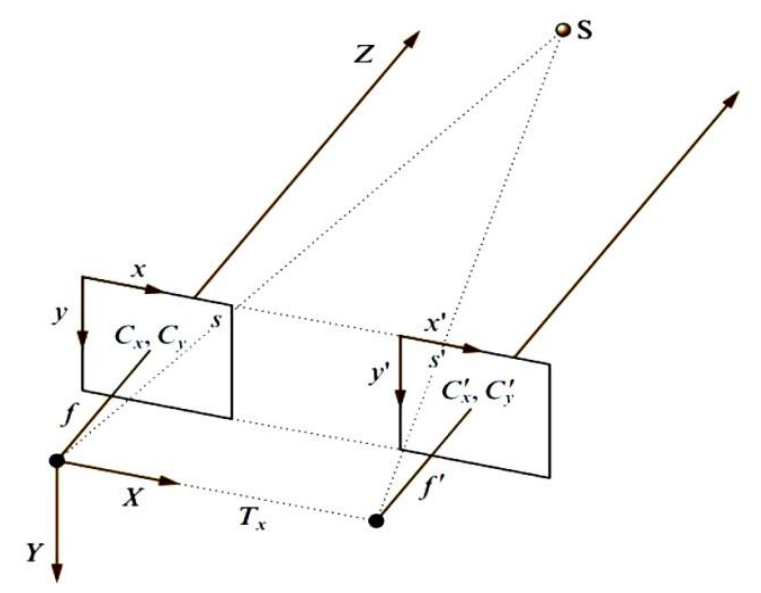

Figure 4. Stereo vision scheme.

(Maryum F. Ahmed, 2006)

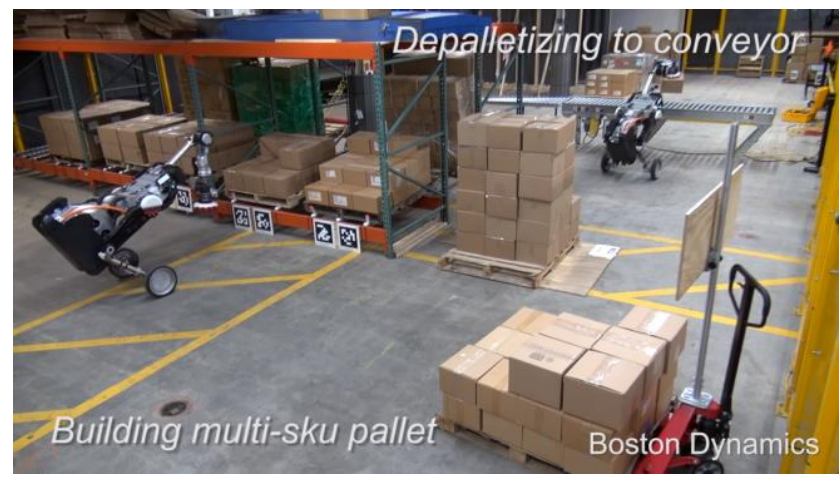

Figure 5. A robotic device manufactured by Boston Dynamics that sorts cargo in a warehouse.

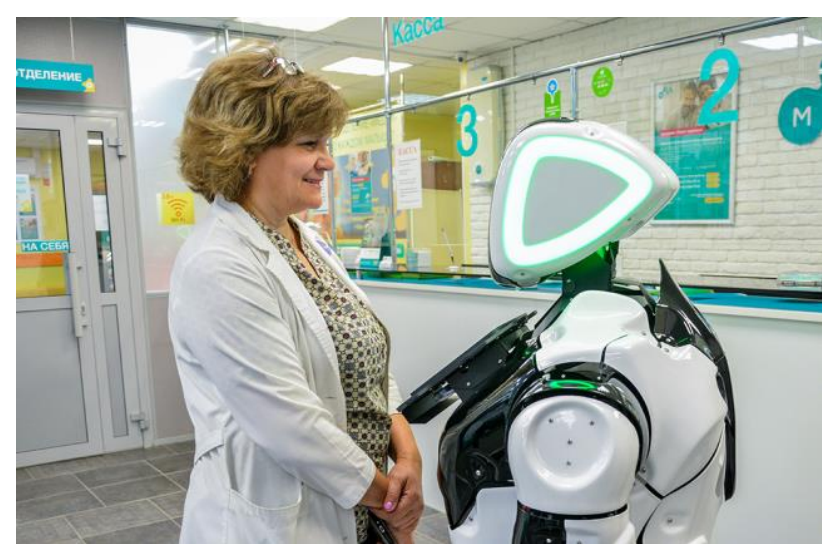

Figure 6. Anthropomorphic robot, Promobot Medical Assessor.

With the help of the technologies presented above, the hardware of a mobile robot can generate a map of the area and build a route not only for one mobile robot, but also for a group of robots (Gradetsky et al., 2011) performing a joint operation task (Figure 7).
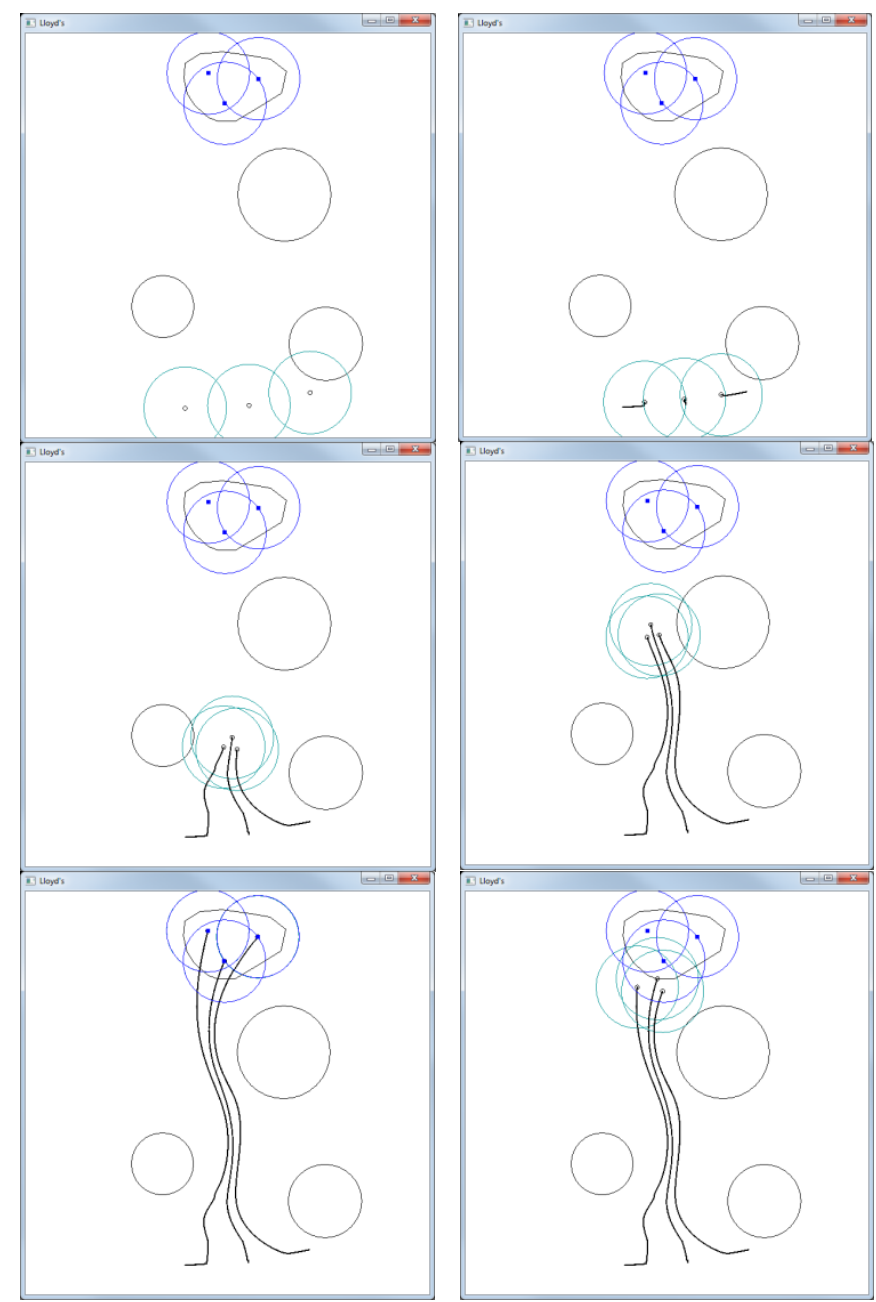

Figure 7. Computer modeling of trajectory planning of mobile robots' group (Gradetsky et al., 2011). 
In order to implement complete coverage of the processed area a special mathematical model for robots' motion is needed. It should also consider and ensure specific distance between robots. Dynamic and static obstacles should be avoided by usage of specific robots' behavior algorithms, which also ensure safety for humans who are present next to robots.

Successful solution of navigation task for group of robots should implement each robot's motion along with target velocity and positioning. Besides requested precision of positioning and velocity there exist restrictions for time for data processing. In that case control system functioning undermine processing of incoming data before new portion of data is given; this is called real-time mode (Dyachenko, 2012).

Therefore it is emerging to unload communication channel, which links robots within their group, and hence to perform calculations on-board of robots. In order to achieve high speed of decision making within control system a special knowledge base should be realized. The latter should incorporate heuristic algorithms to reduce solution of some tasks in some specific situations (Das, 2002)

\section{THE RESULTS}

The priority tasks of robotic support measures to reduce the risk of infection of medical personnel in infected locations are analyzed and the variant of the rational configuration of specialized robots and the directions of their cooperation in the group. As one of the fundamental requirements, the achievement of a sufficiently high level of adaptively of the RSM to the working environment of medical personnel and the known level of autonomy, which allowed to determine the significant role of technical vision systems (VS) in different functionality of specialized robots. It is shown that using VS is the best way to meet the requirements of identification of environment objects when navigating a group of mini-robots with prevention collisions and increasing safety of operations in closed premises together with medical personnel. In the "tracking contacts" mode, VS gives the opportunity (based on interfaces with the temperature measurement means and wearable devices for navigation and finding locations) to perform subsequent epidemiological investigation with use the risk levels data, depending on the individual visits of problematic locations, and contacts with infected persons in latent stage of disease or with persons in which PCR-tests showed hidden (without expressed clinical symptoms) the COVID-19.

The discussion of the results shows that, based on the presented review and analytical material, it is possible to identify priority directions of production of promising samples of the PMS, taking into account the folding social order for improving infectious loads on medical personnel and possible mass demand in this regard, the means of robotic support of the most important and resource intensive works, in particular, to disinfection of closed premises and detection of pathogenes in their air environment. Multifunctional approach to design and using of VSs seems to be the most preferred for unification of solutions when reaching different functionality of robots in the group (Chernousko et al., 2018; Gradetsky et al., 2020; Chirov et al., 2017).

Mini-robots, that are equipped with VS, have a number of advantages compared to robots of big size, even equipped with a full set of sensors and means for autonomous operation: large mobility due to small sizes, significantly lower power requirements and capacity of onboard batteries, low requirements for engine power's, and as a consequence of all this, the lower price for a single product. This approach will allow for the medical personnel and other staff of the first line of health care system to get sufficient number of highly demanded robotic means, which will ensure that the high reliability of operations performed, and will allow not to expose the staff to additional risks of infection in the control of infection cases and disinfection of premises.

Inclusion of VS as the mandatory component of robotic products with additional restrictions on weight and dimensions parameters can provide good ability for passage in a difficult configurable environment of closed premises, ensure timely updating of electronic maps of premises, using elements of geoinformation systems technology to build marking with help of medical personnel of the most dangerous places of infection, identified foci, localization of hazardous objects and localization of patients. Interaction in a group with using the multi-form composition of the tools, may be based on using the VS to coordinate the work and safety of movement in a set, that significantly extends the functionality of each RMS and contributes to a more successful their integration into the human society, as well as adaptation to the infrastructure of specific medical institutions, which are generally variety in their characteristics.

\section{CONCLUSION}

The reduction of personnel Reserve is a limiting factor for sustainable functioning of the health system in its most critical applications, and in this regard, it is necessary to pay close attention to this issue. At the same time, risk of infection can be reduced if such procedures as disinfection of premises, fence and transportation of samples, delivery to consumers of results of their analysis, remote instrumental measurement of health indicators, etc., may be executed by RMSs. As known, the main purpose of robots - is to reduce the workload on staff and eliminate the accompanying danger when the work is monotonous, prolong and dangerous for humans health and activities. Our own research is based on the development of mobile autonomous robots application in the group (Gradetsky et al., 2020) and follow conceptual approaches outlined in the work (Chernousko et al., 2018).

The medical community has the priority task to form a realistic vision of the most acute needs in robotic assistance for security of work in the conditions of risk of infection and reach consensus with developers and manufacturers (of robotics and auxiliary components) regarding acceptable cost with sufficient functionality and opportunities in the shortest possible time to satisfy the demand for RMS in the overwhelming mass of medical institutions involved in the protection the population, and to improve the protection of medical personnel from COVID-19 epidemic's risks.

\section{ACKNOWLEDGEMENTS}

The present work was supported by the Ministry of Science and Higher Education within the framework of the Russian State Assignment under contract No. AAAA-A20-120011690138-6. 


\section{REFERENCES}

Gradetsky V. G., Ermolov I. L., Knyazkov M. M., Lapin B. S., Semenov E. A., Sobolnikov S. A., Sukhanov A. N. Transport group control system of unmanned ground vehicles for various soils// Robotics and technical Cybernetics, 2020. Vol. 8, No. 1, Pp. 61-71. DOI: https://doi.org/10.31776/RTCJ.8107.

Chernousko F. L., Bolotnik N. N. and Gradetsky V. G. Mobile robots: research, development, prospects 2018. http://www.ras.ru/news/shounews.aspx?id=f5c75bcf-2fa5-40e6b06/ (In Rus.) (accessed 12.12.2020).

Chirov D., Chertova O., Potapchuk T. Methods of Study Requirements for the Complex Robotic Vision System// SPIIRAS Proceedings 2(51):152 DOI: 10.15622/sp.51.7.

Chirov D., Chertova O., Potapchuk T. Methods of Study Requirements for the Complex Robotic Vision System// SPIIRAS Proceedings 2(51):152 DOI: 10.15622/sp. 51.7.

Tavakoli, M. (2020). Robotics For COVID-19: How Can Robots Help Health Care in the Fight Against Coronavirus. / M. Tavakoli, J. Carriere, A. Torabi // March 2020. Project: Robotics for COVID-19. URL: https://www.researchgate.net/publication/340270845_Robotics_ For_COVID-

19_How_Can_Robots_Help_Health_Care_in_the_Fight_Again st_Coronavirus

Smirnov A.V., Bezzubcev A.U. Avoiding obstacles with mobile technical means using stereo vision// SOFTWARE SYSTEMS: THEORY AND APPLICATIONS, ISSN 2079-3316 №4 (31), 2016, p. 331-346.

Ryan G., Roelofs R. Simultaneous Localization and Mapping, Swarthmore College. Dept. of Engineering, 2013, URL: http: //thesis.haverford.edu/dspace/handle/10066/11713

Gradetsky V.G., Ermolov I.L., Knyazkov M.M., Sobolnikov S.A. Construction of mobile communication networks based on ground-based autonomous mobile robots// Mechatronics, Automation, Control, No. 11, 2011. P. 126-129

Maryum F. Ahmed. Development of a stereo vision system for outdoor mobile Robots. Abstract of Thesis Presented to the Graduate School of the University of Florida in Partial Fulfillment of the Requirements for the Degree of Master of Science. 2006. pp 78.

Das A.K. et al. A vision-based formation control framework // IEEE transaction on robotics and automation. 2002. Vol. 18(5). P. 813-825.

Dyachenko A. A. The Problem of formation of the system in the UAV group // Proceedings of the Southern Federal University. Technical science. 2012. Vol. 128. N 3. In Russian

Das A.K. A vision-based formation control framework // IEEE transaction on robotics and automation. 2002. Vol. 18(5). P. 813-825. 